\title{
Isotropic-Nematic Density Inversion in a Binary Mixture of Thin and Thick Hard Platelets
}

\author{
H. H. Wensink, G. J. Vroege,* and H. N. W. Lekkerkerker \\ Van't Hoff Laboratory for Physical and Colloid Chemistry, Debye Institute, Utrecht University, Padualaan 8, \\ 3584 CH Utrecht, The Netherlands
}

Received: February 14, 2001

\begin{abstract}
We study the phase behavior of a binary mixture of thin and thick hard platelets, using Onsager's second virial theory for binary mixtures in the Gaussian approximation. Higher virial terms are included by rescaling the excluded volume part of the Onsager free energy using a modified form of the Carnahan-Starling free energy for hard spheres (Parsons' approach). Our calculations provide a simple explanation for the isotropicnematic $(\mathrm{I}-\mathrm{N})$ density inversion, as experimentally observed in systems of polydisperse gibbsite platelets by Van der Kooij et al. (J. Phys. Chem. B 2001, 105, 1696). In these systems, a nematic upper phase was found to coexist with an isotropic bottom phase. We confirm the original conjecture of the authors, which states that the phenomenon originates from a pronounced fractionation in thickness between the phases, such that the thick platelets are largely expelled from the nematic phase and preferentially occupy the isotropic phase. Our calculations show that the inverted state is found in a major part of the $\mathrm{I}-\mathrm{N}$ coexistence region. In addition, a nematic-nematic demixing transition is located at sufficiently high osmotic pressures for any thickness ratio $L_{2} / L_{1}>1$. The $\mathrm{N}-\mathrm{N}$ coexistence region is bounded by a lower critical point which shifts toward lower values as the thickness ratio is increased. At high thickness ratios $\left(L_{2} / L_{1}>3.3\right)$, a triphasic coexistence is found at which two nematic phases coexist with an isotropic phase. We show that the demixing transition is driven by a small $Q(L / D)$ contribution to the excluded volume entropy.
\end{abstract}

\section{Introduction}

Dispersions of hard rod- or platelike colloidal particles have been known to exhibit a spontaneous transition from an isotropic (I) phase, in which the particles are randomly orientated, to an orientationally ordered nematic $(\mathrm{N})$ phase. The physical basis for understanding the phase behavior of anisometrical particles has been described in the classic work of Onsager. ${ }^{1}$ In that paper, Onsager formulated the statistical mechanics of the problem and showed that the transition can be explained on the basis of repulsive interactions between the particles. On the two- particle level, these interactions are represented by the second virial term in an expansion of the free energy of the system. Onsager's theoretical approach was originally inspired by experimental observations of $\mathrm{I}-\mathrm{N}$ phase separation in suspensions of rodlike (tobacco mosaic virus) particles ${ }^{2}$ as well as platelike clay particles. ${ }^{3}$ Numerous other experimental investigations, starting with the work on vanadium pentoxide, ${ }^{4}$ have confirmed the validity of Onsager's theory for suspensions of repulsive rodlike particles. However, experimental evidence of an $\mathrm{I}-\mathrm{N}$ transition in systems of platelike particles is rather limited, being restricted to the observation of an $\mathrm{I}-\mathrm{N}$ transition in suspensions of clay particles by Langmuir in $1938 .^{3}$

Recently, an alternative model system for platelike colloids has been developed, consisting of sterically stabilized gibbsite platelets..$^{5}$ The system exhibits an $\mathrm{I}-\mathrm{N}$ transition at densities that are in fair agreement with computer simulations for hard platelets. An important feature of the gibbsite platelets however is their polydispersity; i.e., the particles differ in size and shape.

The influence of polydispersity on the phase behavior of rodand platelike colloids is an important factor in the interpretation

* To whom correspondence should be addressed. Fax: +31-302533870 E-mail: G.J.Vroege@chem.uu.nl.

10.1021/jp0105894 CCC: $\$ 20.00$ of experimental results, as already pointed out by Onsager in his original paper. ${ }^{1}$ Calculations on binary mixtures of long and short rods ${ }^{6}$ within Onsager's approach have revealed some interesting phenomena such as the fractionation effect (with the longer rods going preferentially to the nematic phase), a widening of the biphasic gap, a reentrant phenomenon, and the possibility of triphasic and nematic-nematic equilibria. ${ }^{7}$

The gibbsite systems, developed by Van der Kooij, display a very broad size distribution in both diameter and thickness. The polydispersity $\sigma$ was found to be approximately $25 \%$ for both diameter and thickness. ${ }^{5}$ Until recently, the effect of polydispersity in the platelets' thickness was considered to be far less important than the polydispersity in diameter, since the thickness hardly contributes to the excluded volume between two platelets and thus cannot have a significant influence on the phase behavior. However, a recent experimental study by Van der Kooij et al. ${ }^{8}$ has shown that polydispersity in thickness can have a considerable influence on the phase behavior of platelike colloids.

In these experiments, the phase behavior of suspensions of gibbsite platelets with a particularly broad distribution in thickness was investigated. ${ }^{8}$ The polydispersity in thickness, although difficult to determine accurately, was estimated at $50 \%$. The high polydispersity is caused by the presence of a significant number of very thick platelets, as observed on transmission electron microscopy (TEM) micrographs of the gibbsite samples. The suspensions show an $\mathrm{I}-\mathrm{N}$ phase separation over a wide range of particle concentrations. However, a remarkable phenomenon was observed; in a major part of the coexistence region a nematic upper phase was found to coexist with an isotropic bottom phase (Figure 1), which implies that the nematic phase has a lower mass density than the coexisting isotropic phase. Henceforth, we will refer to this phenomenon as the $I-N$ density 


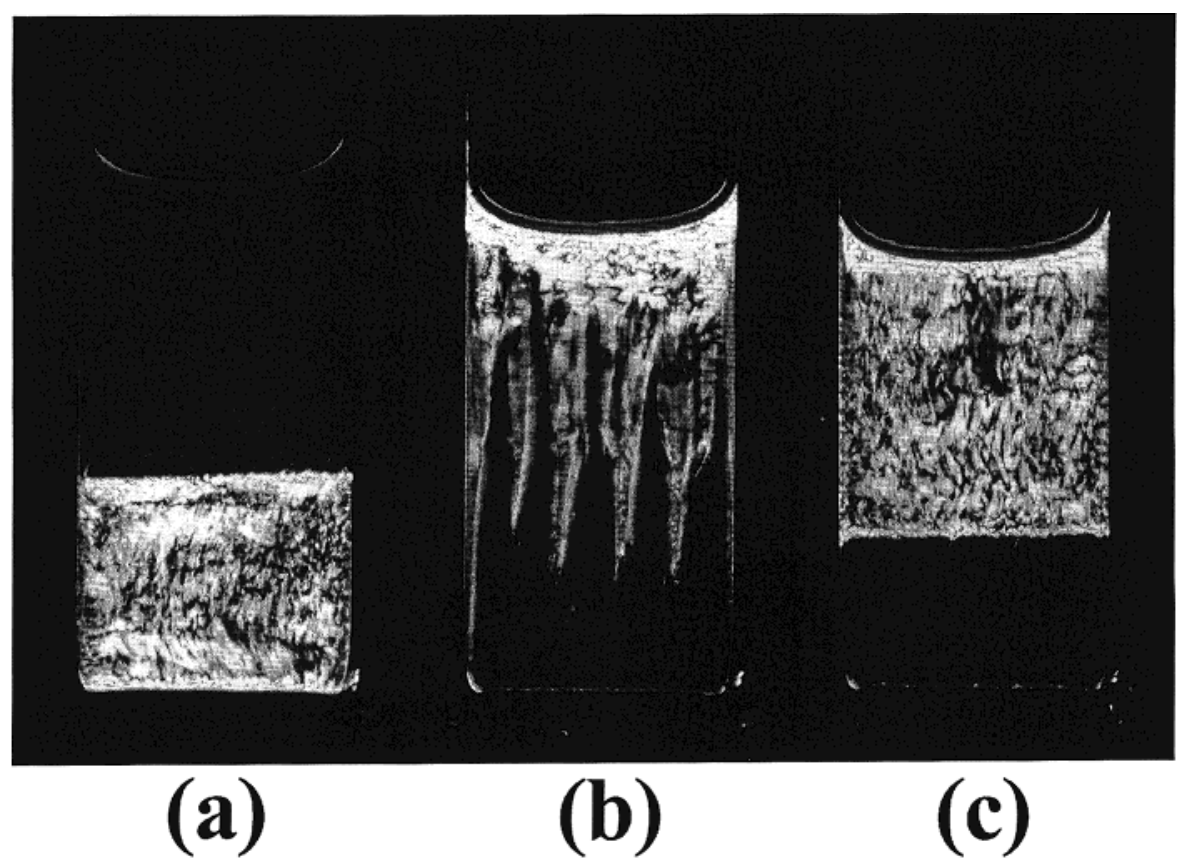

Figure 1. Samples of sterically stabilized gibbsite platelets after I-N phase separation as observed between crossed polarizers. Volume fraction of the samples vary from (a) $\phi=0.22$ to (b) $\phi=0.24$ to (c) $\phi=0.25$. Reprinted from ref 8 . Copyright 2001 American Chemical Society.

inversion. It is argued that this anomalous behavior is related to the platelets' considerable polydispersity in thickness. To explain the underlying mechanism, two features are mentioned by the authors. On one hand, there is a difference between the number densities of the coexisting phases; the total number density of platelets in the nematic phase will in general be higher than that in the coexisting isotropic phase, and consequently, the nematic phase will be more dense than the isotropic phase. On the other hand, there is clear evidence of fractionation in thickness between the coexisting phases, such that the thick platelets (i.e., the largest particles) accumulate in the isotropic phase, thus increasing the mass density of the isotropic phase relative to that of the nematic phase. The authors conjecture that an I-N density inversion occurs when the fractionation is strong enough to overcome the difference between the number densities of the coexisting phases.

Our objective in this paper is to study the phase behavior of a binary mixture of thin and thick hard platelets using Onsager's theory. We show that our calculations indeed account for a significant fractionation effect as well as a density inversion in the $\mathrm{I}-\mathrm{N}$ coexistence region, thus confirming the conjecture of Van der Kooij et al.

Within our theoretical approach, we also locate a nematicnematic demixing transition. We show that a stable demixing transition occurs irrespectively of the thickness ratio. At high ratios, an associated triphasic equilibrium is found. Recently, a similar demixing transition was found in binary mixtures of thin and thick hard rods, ${ }^{9-11}$ although it was found to be stable only at sufficiently high thickness ratios $(\gtrsim 4)$. There, even a stable isotropic-isotropic demixing transition could be located (see also ref 12). We will not pursue this issue in the present paper.

\section{Theory}

Onsager already pointed out that the second virial approach, although valid for infinitely thin needles, cannot be justified for infinitely thin disks. The reason for this is that disks, being two-dimensional objects, have a nonzero probability of intersection and thus a finite excluded volume even at zero thickness. The relative importance of three-body interactions in terms of the ratio $B_{3} / B_{2}^{2}$ (with $B_{3}$ the third virial coefficient) has been estimated by Onsager $^{1}$ at $Q(1)$. More accurate predictions were obtained from computer simulations, ${ }^{13}$ giving $B_{3} / B_{2}{ }^{2} \approx 0.51$ for disks with aspect ratio $L / D=0.1$. These results clearly indicate that Onsager's approach of truncating the free energy after the second virial coefficient cannot be justified (quantitatively) for systems of platelets. To account for the effect of higher-order correlations between particles, we use Parsons' approach ${ }^{14}$ here to incorporate higher virial terms into the Onsager free energy, albeit in an approximate manner. This approach, based on the so-called decoupling approximation in which orientational and translational degrees of freedom are treated separately, comprises a rescaled form of the Carnahan-Starling free energy for hard spheres to describe the (excluded volume) interactions between the anisometrical particles. Recently, Camp and others ${ }^{15,16}$ have shown that the phase behavior of a mixture of hard rods and plates could be described rather successfully within the Parsons approach, showing improved agreement with computer simulation over the Onsager theory. The agreement with simulations was shown to be within $10 \%$.

In this section we present an analytical theory based on the approximate Gaussian trial orientation distribution function (ODF) as formulated by Odijk et al., ${ }^{17}$ which is a simplified version of the trial ODF used by Onsager. ${ }^{1}$ For bidisperse systems of rods with different lengths, the Gaussian ODF successfully explained features such as the fractionation effect, the widened biphasic gap, ${ }^{17}$ and, somewhat later, the existence of triphasic and nematic-nematic equilibria. ${ }^{18} \mathrm{~A}$ recent analysis by van Roij $^{19}$ based on elaborate numerical calculations of the exact high-density ODF essentially confirmed all conclusions of ref 18, thus emphasizing the virtues of the Gaussian approximation.

First, we will give a description of the Onsager formalism for binary mixtures of platelike particles. After that, the Parsons approach will be explained in more detail.

2.1. Onsager Theory. We consider a binary mixture of hard platelets of species $j=1$ or 2 with length (thickness) $L_{j}$ and common diameter $D$ in a macroscopic volume $V$. For the sake of definiteness, we denote the thicker platelets by subscript 2 , 
so that the composition variable $x=N_{2} /\left(N_{1}+N_{2}\right)$ is the mole fraction of the thick platelets. Note that, unlike for a slender rod, the aspect ratio $L / D$ of a thin platelet is a small parameter.

In the case of platelike particles, Onsager described the nematic phase of a dilute solution of platelets in terms of the ODF, $f_{j}(\theta)$, describing the distribution of the angles between the normal to the platelet of type $j$ and the nematic director. The ODF must be normalized according to $\int f_{j}(\Omega) \mathrm{d} \Omega \equiv 1$, where $\Omega$ is the solid angle of the platelet's normal vector. In the isotropic state, all orientations are equally probable, which implies $f_{\text {iso }} \equiv 1 / 4 \pi$.

The formation of an isotropic state (with $f_{j}$ constant) or a nematic state (with $f_{j}$ a peaked distribution) is caused by a competition between orientational entropy (favoring the isotropic state) and excluded volume entropy (favoring the nematic state). Onsager ${ }^{1}$ defined $\sigma_{j}$ as a measure of the (negative of the) orientational entropy

$$
\sigma_{j} \equiv \int f_{j}(\theta) \ln \left[4 \pi f_{j}(\theta)\right] \mathrm{d} \Omega \quad j=1,2
$$

which has its minimum $\left(\sigma_{j}=0\right)$ in the isotropic state but increases as the orientational entropy decreases. In the second virial approximation, the interactions between hard particles may be expressed as an excluded volume entropy depending on the excluded volume between two particles. Onsager gives us the following expression for the excluded volume between two platelets (i.e., circular disks) with thicknesses $L_{j}$ and $L_{k}$ as a function of their mutual angle $\gamma$ :

$$
\begin{aligned}
& v_{\text {excl }}(\gamma)=\frac{\pi}{2} D^{3} \sin \gamma+ \\
&\left(L_{j}+L_{k}\right) D^{2}\left\{\frac{\pi}{4}+E(\sin \gamma)+\frac{\pi}{4}|\cos \gamma|\right\}+\theta\left(\mathrm{L}^{2} D\right)
\end{aligned}
$$

where $E(k)$ is the complete elliptic integral of the second kind. For the isotropic phase, the excluded volume can be readily calculated using the isotropic average $\langle\langle E(\sin \gamma)\rangle\rangle_{\text {iso }}=\pi^{2} / 8$ (see ref 1)

$$
v_{\text {excl, iso }}=\frac{\pi^{2}}{8} D^{3}+\left(L_{j}+L_{k}\right) D^{2}\left\{\frac{\pi^{2}}{8}+\frac{3 \pi}{8}\right\}+\theta\left(L^{2} D\right)
$$

Note that the leading order term does not depend on the thickness $L$ so that the $Q(L / D)$ term must be included to account for the different thicknesses. A measure of the average excluded volume interaction between platelets of types $j$ and $k$ is given by the average of its angular dependence ${ }^{1}$

$$
\rho_{j k} \equiv \iint \frac{v_{\text {excl }}(\gamma)}{v_{\text {excl,iso }}^{0}} f_{j}(\theta) f_{k}\left(\theta^{\prime}\right) \mathrm{d} \Omega \mathrm{d} \Omega^{\prime}
$$

where $v_{\text {excl,iso }}^{0}=D^{3} \pi^{2} / 8$ is the average excluded volume between two randomly orientated platelets with zero thickness. The definition of $\rho_{j k}$ is slightly different from the one used by Onsager, since we only retain the leading order term $v_{\text {excl,iso }}^{0}$ in the denominator instead of the full expression (3). This leads to $\rho_{j k}^{\text {iso }}=1+\theta(L / D)$ whereas in Onsager's theory $\rho^{\text {iso }} \equiv 1$, by definition. Note that both definitions are equivalent up to leading order. Substituting (3) into (4) yields for the isotropic phase

$$
\rho_{j k}^{\text {iso }}=1+\frac{L_{j}+L_{k}}{D}\left(1+\frac{3}{\pi}\right)+\Theta\left(L^{2} / D^{2}\right)
$$

Note that the second contribution is on the order of $L / D$ smaller than the leading order term. We can express the total Helmholtz free energy (in units $k_{\mathrm{B}} T$ per particle) of a binary mixture within the second virial approximation in terms of $\sigma_{j}$ and $\rho_{j k}$, leading to

$$
\begin{aligned}
& \frac{\beta F}{N_{1}+N_{2}} \sim c s t-1+\ln c+(1-x) \ln (1-x)+x \ln x+ \\
& \quad(1-x) \sigma_{1}+x \sigma_{2}+c\left[(1-x)^{2} \rho_{11}+2 x(1-x) \rho_{12}+x^{2} \rho_{22}\right]
\end{aligned}
$$

where $\beta=1 / k_{\mathrm{B}} T$ in terms of Boltzmann's constant $k_{\mathrm{B}}$ and the absolute temperature $T$. Furthermore, $c$ is the total number density of platelets rendered dimensionless by relating it to $v_{\text {excl,iso }}^{0}$ in the following way:

$$
c=\frac{1}{2} v_{\text {excl,iso }}^{0} \frac{N_{1}+N_{2}}{V}=\frac{\pi^{2}}{16} D^{3} \frac{N_{1}+N_{2}}{V}
$$

The last term in (6) can be identified as the (dimensionless) second virial coefficient $\tilde{B}_{2}$ multiplied by the concentration $c$. Note that $c \tilde{B}_{2}$ constitutes the excess part of the free energy, which accounts for the interactions between the hard particles. Using (5), together with the isotropic value, $\sigma_{j} \equiv 0$, we get the following expression for the free energy in the isotropic phase:

$$
\begin{array}{r}
\frac{\beta F_{\text {iso }}}{N_{1}+N_{2}} \sim c s t+(\ln c-1)+(1-x) \ln (1-x)+x \ln x+ \\
c\left\{1+\left(2+\frac{6}{\pi}\right)\left[(1-x) \frac{L_{1}}{D}+x \frac{L_{2}}{D}\right]\right\}
\end{array}
$$

In the nematic phase, matters are more complicated since the ODF is no longer a constant but a sharply peaked function. The excluded volume entropy is now given by

$$
\begin{array}{r}
\rho_{j k}^{\mathrm{nem}}=\frac{4}{\pi} \iint|\sin \gamma| f_{j}(\theta) f_{k}\left(\theta^{\prime}\right) \mathrm{d} \Omega \mathrm{d} \Omega^{\prime}+\frac{2}{\pi} \frac{L_{j}+L_{k}}{D} \times \\
\iint\left[3-\frac{1}{2} \sin ^{2} \gamma+|\cos \gamma|\right] f_{j}(\theta) f_{k}\left(\theta^{\prime}\right) \mathrm{d} \Omega \mathrm{d} \Omega^{\prime}+ \\
\theta\left[(L / D)^{2}\right]
\end{array}
$$

Here, the following asymptotic expansion of the elliptic integral is used:20

$$
E(\sin \gamma)=\frac{\pi}{2}\left\{1-\frac{1}{4} \sin ^{2} \gamma+Q\left(\sin ^{4} \gamma\right)\right\}
$$

which is valid for very small angles $\gamma$. This approximation is justified when the ODF is a sharply peaked function. Obviously, we must specify the ODF $f_{j}$ to calculate $\rho_{j k}$. As in ref 17 , we use Gaussian trial ODFs with variational parameter $\alpha_{j}$ to describe the angular distribution of the platelets $j$ in the nematic state

$$
f_{j}(\theta) \equiv \begin{cases}\frac{\alpha_{j}}{4 \pi} \exp \left[-\frac{1}{2} \alpha_{j} \theta^{2}\right] & 0 \leq \theta \leq \frac{\pi}{2} \\ \frac{\alpha_{j}}{4 \pi} \exp \left[-\frac{1}{2} \alpha_{j}(\pi-\theta)^{2}\right] & \frac{\pi}{2} \leq \theta \leq \pi\end{cases}
$$

A great advantage of using a Gaussian trial ODF is that $\sigma_{j}$ and $\rho_{j k}$ are now analytically tractable. Substituting (11) in (1) gives us

$$
\sigma_{j} \sim \ln \alpha_{j}-1 \quad j=1,2
$$

for the orientational entropy. For the excluded volume entropy in the nematic phase, we will only retain the leading order terms 
of its asymptotic expansion for large $\alpha_{j}$

$$
\rho_{j k}^{\mathrm{nem}} \sim\left[\frac{8}{\pi}\left(\frac{1}{\alpha_{j}}+\frac{1}{\alpha_{k}}\right)\right]^{1 / 2}+\frac{8}{\pi} \frac{L_{j}+L_{k}}{D}\left[1+Q\left(\alpha_{j}^{-1}, \alpha_{k}^{-1}\right)\right]
$$

Henceforth, we will neglect the $\theta\left(\alpha^{-1}\right)$ contribution in the second term, which is justified for very large values of $\alpha$. Note that this is a crucial step in our calculations, since the $Q(L / D)$ term now becomes independent of $\alpha_{j}$ and thus independent of the width of the angular distribution $f_{j}(\theta)$. An important consequence is that, in our approximation, the orientation of the platelets in the nematic phase is solely determined by the diameter of the platelets, which is identical for both components. It is important to realize that the thickness does not enter into the free energy anywhere else than via the $Q(L / D)$ term in (13). So, we can simplify our calculations considerably by using a single variational parameter, $\alpha=\alpha_{j}=\alpha_{k}$, which holds for both components. The above expression then reduces to

$$
\rho_{j k}^{\text {nem }} \sim \frac{4}{(\pi \alpha)^{1 / 2}}+\frac{8}{\pi} \frac{L_{j}+L_{k}}{D}
$$

Note that the $L / D$ contribution to $\rho^{\text {nem }}$ is simply the excluded volume between two perfectly parallel platelets $(\gamma=0)$ in the nematic phase, $\pi D^{2}\left(L_{j}+L_{k}\right)$, divided by the excluded volume between two randomly oriented platelets with zero thickness in the isotropic phase $\left(v_{\text {excl,iso }}^{0}\right)$. The $L / D$ contribution remains constant up to order $\theta\left(\gamma^{2}\right)$ as can easily be seen from (9) by substituting the asymptotic expressions for the trigonometric functions involved. Inserting (12) and (14) into the Helmholtz free energy (6) and minimizing with respect to $\alpha$ gives

$$
\alpha \sim 4 c^{2} / \pi
$$

independent of the mole fraction $x$. We see that $\alpha$ exhibits a simple $c^{2}$ dependence which is frequently encountered in Onsager's approach. Substituting all expressions back into the free energy (6) yields a simple expression for the free energy in the nematic phase

$$
\begin{aligned}
& \frac{\beta F_{\text {nem }}}{N_{1}+N_{2}} \sim c s t+3 \ln c+\ln \frac{4}{\pi}+x \ln x+ \\
& \quad(1-x) \ln (1-x)+\frac{16}{\pi} c\left[(1-x) \frac{L_{1}}{D}+x \frac{L_{2}}{D}\right]
\end{aligned}
$$

To locate phase transitions, we must know the osmotic pressure and chemical potential of both types of platelets. These are calculated as derivatives of the free energy. In the nematic phase we obtain for the osmotic pressure (in dimensionless notation)

$$
\begin{aligned}
\tilde{\Pi}_{\text {nem }} \equiv-\frac{1}{2} \beta v_{\text {excl,iso }}^{0}\left(\frac{\partial F_{\text {nem }}}{\partial V}\right)_{N_{1}, N_{2}, T} \\
\sim 3 c_{\mathrm{n}}+\frac{16}{\pi} c_{\mathrm{n}}^{2}\left[\left(1-x_{\mathrm{n}}\right) \frac{L_{1}}{D}+x_{\mathrm{n}} \frac{L_{2}}{D}\right]
\end{aligned}
$$

where $c_{\mathrm{n}}$ and $x_{\mathrm{n}}$ refer to the concentration and composition of the nematic phase. The chemical potentials (also in dimensionless notation) can be obtained from

$$
\tilde{\mu}_{j, \text { nem }} \equiv \beta\left(\frac{\partial F_{\text {nem }}}{\partial N_{j}}\right)_{N_{j}, V, T} \quad j=1,2
$$

Expressions for the isotropic phase can be obtained likewise from (8). The excess free energy in the nematic phase is essentially given by terms like

$$
c \rho_{j k}^{\text {nem }} \sim 2+\frac{8}{\pi} c \frac{L_{j}+L_{k}}{D}
$$

Note that the term $c \tilde{B}_{2}$ in the free energy (6) can be obtained from $c \rho_{j k}{ }^{\text {nem }}$ by taking the average over all pairs of components $j k$, using the product of mole fractions $x_{j} x_{k}$ as a weight function. In the leading order term of (14), the $c$ dependence vanishes due to the fact that $\alpha \sim c^{2}$, according to (15). As a result, the leading order contribution to the excess free energy, i.e., for platelets with zero thickness, is simply 2 and thus independent of the concentration. The $Q(L / D)$ term gives an additional contribution which is linear in c. In the osmotic pressure (17), this leads to an additional $c^{2}$ contribution to the common linear term 3c. Hence, the thickness of the platelets will have a considerable influence on the osmotic pressure of a concentrated nematic phase.

We are, in principle, ready to construct the phase diagram by equating the osmotic pressure and the chemical potentials of the isotropic and nematic phases and solve these equations iteratively. However, as we already pointed out in the Introduction, one has to be very careful in adopting Onsager's second virial theory to describe a system of platelike particles since higher virial coefficients will undoubtedly play a role, even at low concentrations. Therefore, to make quantitative comparison with experiments possible, we have to somehow account for the effect of higher virial terms. A method which has proved to be remarkably accurate up to now is to rescale the excess part of the Onsager free energy using a modified form of the Carnahan-Starling excess free energy for hard spheres. This approach, which is known as the Parsons approach, will be discussed in the next section.

2.2. The Parsons Approach. This approach due to Parsons ${ }^{14}$ from the late 1970s yields an extension of the CarnahanStarling expression for the excess free energy of hard spheres to anisometrical particles. The well-known Carnahan-Starling (CS) equation of state gives a very accurate description of a hard-sphere fluid at volume fractions up to the freezing fraction $(\phi \approx 0.5)$. In fact, in that regime, the equation of state is almost indistinguishable from the one obtained from computer simulations. About 10 years later Lee ${ }^{21}$ showed that Parsons' approach gave an accurate description of the isotropic to nematic transition in a system of hard ellipsoidal particles. The success of the approach relies on the incorporation of many higher order interactions, albeit approximately. As an example, for hard spherocylinders with aspect ratio $L / D=5$, the third virial coefficient calculated from Parsons' theory ${ }^{14,22}$ is $B_{3}=0.2972 B_{2}{ }^{2}$ compared to $B_{3}=0.4346 B_{2}{ }^{2}$ from Monte Carlo simulations. ${ }^{23}$

The Parsons approach involves an expression of the excess free energy in terms of the semiempirical Carnahan-Starling excess free energy for hard spheres ${ }^{24}$

$$
f_{\mathrm{CS}}(\phi) \equiv \frac{\beta F_{\mathrm{CS}}^{\mathrm{ex}}}{N}=\frac{\phi(4-3 \phi)}{(1-\phi)^{2}}
$$

where $\phi$ is the volume fraction of hard spheres. For a one-component system of hard anisometrical particles, this free energy is multiplied by the prefactor $\left\langle\left\langle v_{\text {excl }}\right\rangle\right\rangle / 8 v_{0}$, with $v_{0}$ the particle volume and $\left\langle\left\langle v_{\text {excl }}\right\rangle\right\rangle$ the average excluded volume. Note that $\left\langle\left\langle v_{\text {excl }}\right\rangle\right\rangle / 8 v_{0}=1$, in the case of hard spheres. For binary mixtures of anisometrical particles, the prefactor could be rewritten as $\left\langle\left\langle\bar{v}_{\text {excl }}\right\rangle\right\rangle / \bar{v}_{0}$ in terms of the following mole fraction averages: $^{15}$ 


$$
\begin{aligned}
\left\langle\left\langle\bar{v}_{\text {excl }}\right\rangle\right\rangle & =v_{\text {excl,iso }}^{0}\left[(1-x)^{2} \rho_{11}+2 x(1-x) \rho_{12}+x^{2} \rho_{22}\right] \\
& =v_{\text {excl,iso }}^{0} \tilde{B}_{2} \\
\bar{v}_{0} & =(1-x) v_{0,1}+x v_{0,2} \\
\phi & =(1-x) \phi_{1}+x \phi_{2}
\end{aligned}
$$

where $\phi$ is the total volume fraction of particles, related to the dimensionless concentration and composition via

$$
\phi(c, x)=\frac{4}{\pi} c\left[(1-x) \frac{L_{1}}{D}+x \frac{L_{2}}{D}\right]
$$

Having established this, we can write the excess free energy within the Parsons approach as follows:

$$
\begin{aligned}
\frac{\beta F^{\mathrm{ex}}}{N} & =c\left[(1-x)^{2} \rho_{11}+2 x(1-x) \rho_{12}+x^{2} \rho_{22}\right] \tilde{f}_{\mathrm{CS}}(\phi) \\
& =c \tilde{f}_{\mathrm{CS}}(\phi) \tilde{B}_{2}
\end{aligned}
$$

where $\tilde{f}_{\mathrm{CS}}(\phi)=f_{\mathrm{CS}}(\phi) / 4 \phi$. Replacing the excess free energy $c \tilde{B}_{2}$ in (6) by (23) gives us the Onsager-Parsons free energy for a binary mixture of hard platelets. Accordingly, for the isotropic phase, we must replace $c$ by $c \tilde{f}_{\mathrm{CS}}$ in the last term of (8). Recalculation of the osmotic pressure and chemical potentials for the isotropic phase is now straightforward, using the definitions (17) and (18). Recall that the volume fraction depends on $c$ and $x$ via (22) so that the resulting expressions involve additional derivatives of $\tilde{f}_{\mathrm{CS}}$ with respect to $c$ and $x$. For the nematic phase, matters are slightly more complicated because of the minimization step. Hence, minimization with respect to $\alpha$ now yields

$$
\alpha \sim \frac{4}{\pi} c^{2} \tilde{f}_{\mathrm{CS}}^{2}(\phi)
$$

The Onsager-Parsons free energy (denoted by superscript "P")for the nematic phase thus reads

$$
\begin{array}{r}
\frac{\beta F_{\mathrm{nem}}^{\mathrm{P}}}{N_{1}+N_{2}} \sim c s t+3 \ln c+\ln \frac{4}{\pi}+x \ln x+(1-x) \ln (1- \\
x)+2 \ln \tilde{f}_{\mathrm{CS}}(\phi)+\frac{16}{\pi} c \tilde{f}_{\mathrm{CS}}(\phi)\left[(1-x) \frac{L_{1}}{D}+x \frac{L_{2}}{D}\right]
\end{array}
$$

Note the additional $\ln \tilde{f}$ contribution arising from the orientational entropy (12). Expressions for the osmotic pressure and chemical potentials can be obtained similarly from the free energy by straightforward derivations. To facilitate comparison with (17), we will only give the osmotic pressure of the nematic phase

$$
\begin{aligned}
& \tilde{\Pi}_{\mathrm{nem}}^{\mathrm{P}} \sim c_{\mathrm{n}}+\left[2 c_{\mathrm{n}}+\frac{16}{\pi} c_{\mathrm{n}}^{2} \tilde{f}_{\mathrm{CS}}\left\{\left(1-x_{\mathrm{n}}\right) \frac{L_{1}}{D}+x_{\mathrm{n}} \frac{L_{2}}{D}\right\}\right] \times \\
& {\left[1+c_{\mathrm{n}} \frac{\partial \ln \tilde{f}_{\mathrm{CS}}}{\partial c}\right] }
\end{aligned}
$$

Note that the linear contribution $3 c_{\mathrm{n}}$ is retained. However, the presence of $\tilde{f}$ and its derivatives leads to a more complicated $c$ dependence. Similar expressions can be obtained for the chemical potentials and the pressure in the isotropic phase.

\section{Isotropic-Nematic Phase Coexistence: Density Inversion}

We can construct the phase diagram by imposing the standard conditions of equal pressure and chemical potentials in the two
TABLE 1: Typical Dimensions and Aspect Ratios of the Thick and Thin Platelets Used in the Present Calculations ${ }^{31}$

\begin{tabular}{ccccc}
\hline$D(\mathrm{~nm})$ & $L_{1}(\mathrm{~nm})$ & $L_{2}(\mathrm{~nm})$ & $D / L_{1}$ & $D / L_{2}$ \\
\hline 180 & 20 & 45 & 9 & 4
\end{tabular}

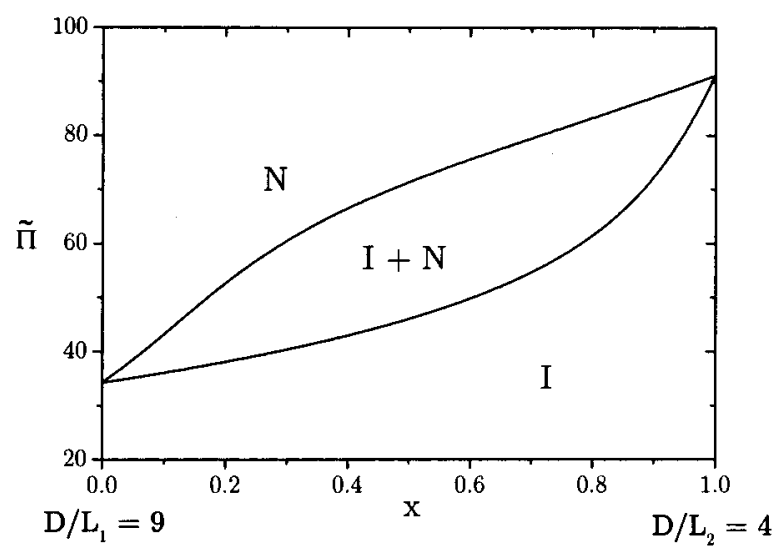

Figure 2. Phase diagram in the $\tilde{\Pi}-x$ plane of a binary mixture of thin platelets $\left(D / L_{1}=9\right)$ and thick platelets $\left(D / L_{2}=4\right)$, calculated from the Onsager-Parsons free energy. Note the significant degree of fractionation between the phases.

coexisting phases, where the pressure and chemical potential follow from the Onsager-Parsons equilibrium free energy. However, we are still left with two important, yet unspecified, parameters: the typical aspect ratios of the thin and thick platelets, $L_{1} / D$ and $L_{2} / D$, respectively. Since it is our primary aim to account for the experimentally observed features, we restrict ourselves to a single combination of aspect ratios, rather than scanning the entire parameter space. We have chosen a particular combination of dimensions for the platelets under consideration, shown in Table 1 . These values should resemble the experimental system, studied by Van der Kooij et al., ${ }^{8}$ in a reasonable way. The corresponding phase diagram is depicted in Figure 2. The diagram clearly displays a considerable degree of fractionation between the coexisting phases. The thick platelets are largely expelled from the nematic phase and prefer to inhabit the isotropic phase instead. Another notable feature of Figure 2 is the strong increase of the equilibrium osmotic pressure at increasing mole fractions, indicating that the isotropic to nematic transition in a pure system of platelets shifts to higher number densities as the thickness is increased.

When we want to study the possibility of an $\mathrm{I}-\mathrm{N}$ density inversion, we have to calculate the total mass density of the isotropic and the nematic phases. The mass density of the phases is linearly proportional to the effective core volume fraction of the platelets

$$
\phi_{\text {core }}=\frac{4}{\pi} c\left[(1-x) \frac{L_{1}}{D} \frac{v_{1}^{\text {core }}}{v_{1}}+x \frac{L_{2}}{D} \frac{v_{2}^{\text {core }}}{v_{2}}\right]
$$

which stems from the fact that, experimentally, the colloidal platelets consist of a (dense) gibbsite $\left(\mathrm{Al}(\mathrm{OH})_{3}\right)$ core surrounded by a grafted polymer layer. The polymer layer approximately has the same density as the solvent in which the platelets are immersed and hence does not contribute to the total density of the platelets. However, the grafted polymer layer does participate in the mutual excluded volume between two platelets. The dimensions given in Table 1 thus apply to the grafted gibbsite platelets. The thickness of the polymer layer has been estimated at $4 \mathrm{~nm},{ }^{8}$ and the ratio of the core volume $v_{\text {core }}$ to the total volume $v$ of the platelet can thus be calculated using the values from Table 1 , giving $v_{1}{ }^{\text {core }} / v_{1} \approx 0.55$ and $v_{2}{ }^{\text {core }} / v_{2} \approx 0.75$. 


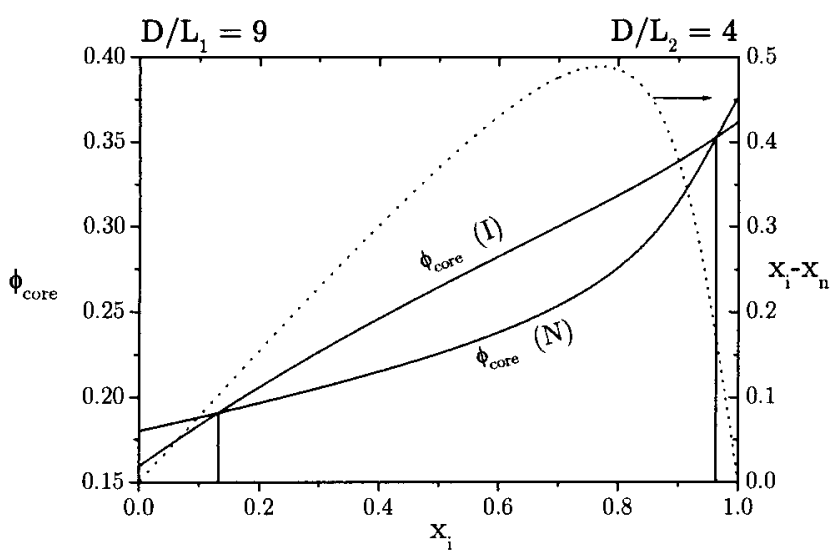

Figure 3. Core volume fraction $\phi_{\text {core }}$ of the coexisting phases versus $x_{\mathrm{i}}$. In the area between the vertical lines the phase densities are inverted; i.e., the isotropic phase is more dense than the nematic phase. On the right axis, the extent of fractionation $\left(x_{\mathrm{i}}-x_{\mathrm{n}}\right)$ is plotted versus $x_{\mathrm{i}}$.

Figure 3 clearly reveals that a density inversion will indeed take place during the $\mathrm{I}-\mathrm{N}$ phase separation. A marked feature is that the area in which the isotropic and nematic phase densities are inverted covers a major part of the phase diagram. Only when the overall mole fraction is close to zero or one, i.e., in the case of an almost pure system of either thin or thick platelets, is fractionation apparently not strong enough to accomplish a density inversion. In these situations, an isotropic upper phase will be found as in the regular cases. Equal phase densities are found at coexisting mole fractions $\left(x_{\mathrm{i}}, x_{\mathrm{n}}\right)=(0.181,0.041)$ and $(0.954,0.747)$, corresponding to $\tilde{\Pi}=37.7$ and 81.2 , respectively.

We can also represent the phase diagram by plotting the core volume fraction versus the concentration, as shown in Figure 4. Recall that the core volume fraction is directly proportional to the mass density of the phases. In this representation, we can draw dilution lines, i.e., straight lines radiating from the origin, along which the overall composition $x$ of the parent system remains constant. From this representation we immediately see that the total number density of platelets in the nematic phase is always higher than the number density in the isotropic phase, irrespective of the slope of the dilution line. This clearly indicates that the density inversion is not stimulated by the difference between the thermodynamic number densities of the coexisting phases and hence must be driven by fractionation. Furthermore, the slopes of the tie lines now indicate the relative densities of the coexisting isotropic and nematic phases, such that, in the case of a negative slope, the inverted state will be found. Note that the evolution of the slopes of the tie lines gives the impression of a "spiral staircase" in which the slopes gradually change from positive to negative back to positive, as one goes from a pure system of thin platelets to a pure system of thick platelets. Another marked feature in Figure 4 is the widening of the biphasic gap, although less pronounced here than in the case of rods with different lengths. ${ }^{6,17}$ The widening of the $\mathrm{I}-\mathrm{N}$ biphasic gap appears to be a typical property of bidisperse (and polydisperse) systems of anisometrical particles ${ }^{25}$ as well as mixtures of different (polydisperse) species, such as rod-plate mixtures ${ }^{26,27}$ and plate-polymer mixtures. ${ }^{28}$

\section{Nematic-Nematic Phase Coexistence}

As mentioned earlier in this paper, the thickness of the platelets has a considerable influence on the osmotic pressure of a concentrated nematic phase, as we can see from (17). Naturally, one may ask whether this can cause the nematic phase
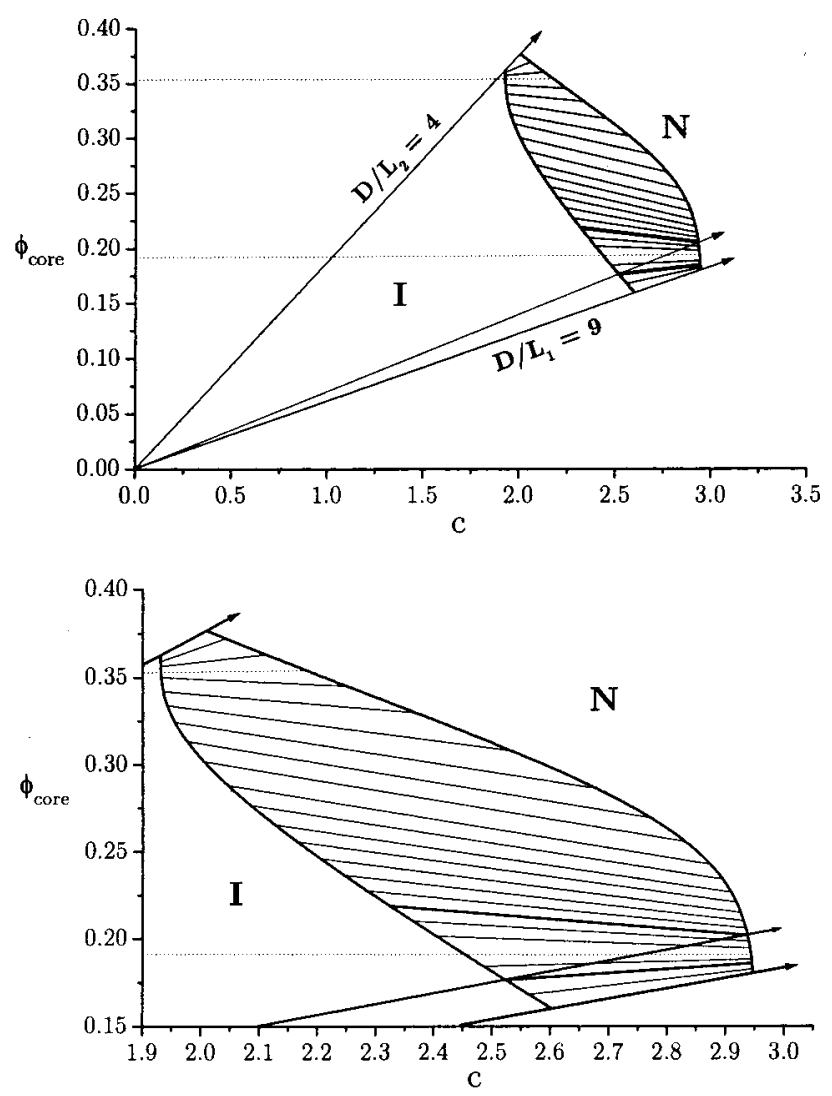

Figure 4. (a, top) Phase diagram in the phase density-concentration $\left(\phi_{\text {core }}-c\right)$ plane. The outer dilution lines correspond to the pure components. Thick lines indicate phase boundaries; thin lines represent tie lines connecting coexisting phases. The horizontal tie lines (dotted lines) denote equal phase densities. In the area between the dotted lines the tie lines have negative slopes, which correspond to the inverted state. (b, bottom) Magnification of the biphasic area.

of a binary mixture of thin and thick platelets to demix into two nematic phases at sufficiently high concentrations.

In this section we intend to study the relation between the thickness bidispersity, quantified by the thickness ratio $L_{2} / L_{1}$, and the topology of the phase diagram, in particular the existence of a nematic-nematic coexistence region. The occurrence of a demixing transition at a particular osmotic pressure can easily be identified by the presence of an instability region (or van der Waals loop) in the chemical potential curve (plotted versus the mole fraction) in which $\partial \mu_{j} / \partial x_{j}<0$. When we study the possible coexistence between two nematic phases, we have to realize that the osmotic pressure and chemical potentials are given by the same expressions $(17,18)$ in both phases. To obtain two different nematic phases, there must be two states, denoted by I and II, with different $c_{\mathrm{n}}$ and/or $x_{\mathrm{n}}$ values, having the same osmotic pressure and chemical potential.

We will proceed in the following way: The aspect-ratio of the thick platelets will be fixed at 4 while the aspect ratio of the thin platelets is subject to variation. This implies that we increase the degree of bidispersity by making the thin platelets thinner while keeping the thickness of the thick platelets fixed. In Figure 5, the resulting phase diagrams are depicted for different values of $D / L_{1}$.

A remarkable feature is that the nematic-nematic transition is always present, irrespective of the thickness ratio $L_{2} / L_{1}(>1)$. Thus, even systems with a low degree of bidispersity (i.e., $L_{2} /$ $L_{1}$ close to 1 ) exhibit a nematic-nematic demixing transition, albeit at very high osmotic pressures. For instance, in our experimentally considered system $\left(D / L_{1}=9, D / L_{2}=4\right)$, a 

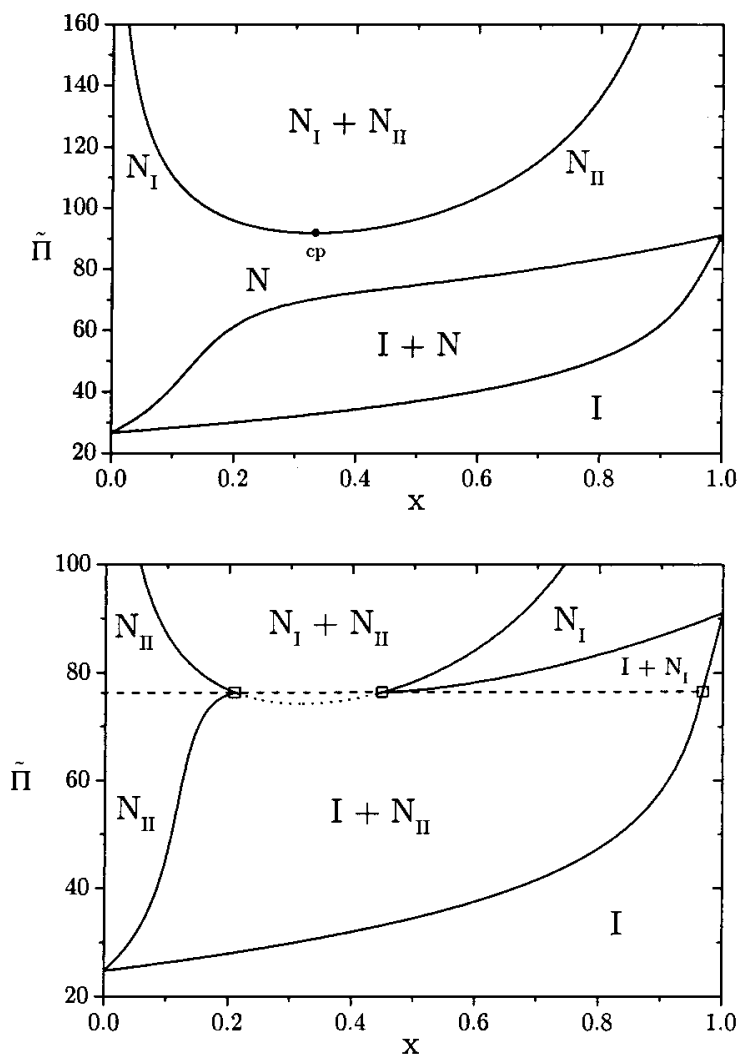

Figure 5. (a, top) Phase diagram in the $\tilde{\Pi}-x$ plane of a binary mixture of thin platelets, $D / L_{1}=13$, and thick platelets, $D / L_{2}=4\left(L_{2} / L_{1}=\right.$ 3.25). The nematic-nematic coexistence region is bounded by a lower critical point (cp). (b, bottom) Same as (a), for $D / L_{1}=15$ and $D / L_{2}=$ $4\left(L_{2} / L_{1}=3.75\right)$. Full curves denote stable phase boundaries, while the dotted curve represents a metastable phase boundary. The $\mathrm{I}-\mathrm{N}_{\mathrm{I}}-$ $\mathrm{N}_{\text {II }}$ triple point is indicated by $\square$.

nematic-nematic critical point is located at a coexistence pressure $\tilde{\Pi}=208$. Note that several other (liquid) crystalline phases may come into play at these high pressures. When the thickness ratio is increased, the critical point shifts to lower values of the osmotic pressure, until at $L_{2} / L_{1} \approx 3.3$ the $\mathrm{N}-\mathrm{N}$ and $\mathrm{I}-\mathrm{N}$ coexistence regions start to overlap, giving rise to a triple point at which two nematic phases $\left(\mathrm{N}_{\mathrm{I}}\right.$ and $\left.\mathrm{N}_{\mathrm{II}}\right)$ coexist with an isotropic phase (I) (Figure 5b).

Let us now elaborate on the nematic-nematic demixing transition for a while and try to gain more insight into the underlying mechanism. A convenient way to study the mechanism behind a demixing transition is to construct the Gibbs free energy of the nematic phase and investigate the behavior of the individual entropic contributions. For the sake of simplicity, we will use the expressions from the Onsager free energy (16) here, rather than the elaborate formulas obtained from the Parsons rescaling step. The Gibbs free energy (in units of $k_{\mathrm{B}} T$ per particle) is given by

$$
g(\tilde{\Pi}, x) \equiv \frac{\beta F}{N}+\tilde{\Pi} c^{-1}(\tilde{\Pi}, x)
$$

where the concentration $c$ is now a function of the osmotic pressure $\tilde{\Pi}$ and the mole fraction $x$. This function can readily be obtained by inverting the expression for the osmotic pressure in the nematic phase (17), which is simply quadratic in $c$. The individual entropic contributions are given by

$$
g_{\text {mix }} \sim(1-x) \ln (1-x)+x \ln x
$$

$$
\begin{gathered}
g_{\text {or }} \sim 2 \ln c(\tilde{\Pi}, x)+\ln \frac{4}{\pi}+1 \\
g_{\text {ex }} \sim 2+\frac{32}{\pi} c(\tilde{\Pi}, x)\left[(1-x) \frac{L_{1}}{D}+x \frac{L_{2}}{D}\right]
\end{gathered}
$$

where $g_{\text {mix }}, g_{\text {or }}$, and $g_{\text {ex }}$ refer to mixing entropy, orientational entropy, and excluded volume entropy, respectively. The subscripts for the nematic phase are left out for notational convenience. Note that the translational entropy contribution $(\sim \ln c)$ is omitted here, since it has essentially the same dependence as the orientational part.

It is advantageous to rescale the Gibbs free energy by subtracting the chemical potentials of the pure components, weighed by their mole fractions

$$
g^{\prime}(\tilde{\Pi}, x) \equiv g(\tilde{\Pi}, \mathrm{x})-\left[(1-x) \mu_{1}^{0}(\tilde{\Pi})+x \mu_{2}^{0}(\tilde{\Pi})\right]
$$

Obviously, the same rescaling procedure can be applied to the individual entropic contributions. The motivation behind the rescaling of $g$ is to uncover the exact curvature of the free energy by subtracting the dominant linear trend. Note that the rescaling is fully justified since only a linear contribution is subtracted from the Gibbs free energy. Hence, the usual double tangent construction for the determination of the coexistence compositions can still be applied here. We can now plot the rescaled Gibbs free energy versus the mole fraction for any particular osmotic pressure and combination of aspect ratios. Recall that the osmotic pressure should remain fixed upon variation of $x$. Figure 6 clearly reveals that the demixing transition originates from a competition among translational entropy, mixing entropy, and orientational entropy on one hand (all favoring the mixed state) and excluded volume entropy on the other hand (favoring demixing). At sufficiently high osmotic pressures, the latter contribution will become dominant and demixing occurs.

\section{Discussion}

Our calculations based on the Gaussian approximation provide us with a fairly simple interpretation of the isotropic-nematic density inversion, as observed in experimental systems of polydisperse gibbsite platelets. ${ }^{8}$ It appears that this phenomenon indeed originates from a pronounced fractionation with respect to thickness between the isotropic and the nematic phases, as already suggested by van der Kooij et al. ${ }^{8}$ Hence, an isotropicnematic density inversion can only be accomplished when the fractionation is strong enough to overrule the difference between the coexistence number densities of the isotropic and the nematic phases, for which we have shown that $c_{\mathrm{i}}<c_{\mathrm{n}}$, irrespective of the overall composition. In this respect, we stress the importance of the Parsons rescaling procedure. It is obvious that the possibility of a density inversion strongly depends on the difference between the coexistence densities (miscibility gap) as found from the solutions of the coexistence equations. It turns out that including higher virial terms by means of Parsons' approach leads to a significant narrowing of the miscibility gap and therefore strongly promotes fractionation to dominate, giving rise to an inversion of densities.

It is a known feature that the Onsager theory overestimates the miscibility gap, predicting a strong first-order phase transition $\left(\Delta\left(N D^{3} / V\right)>1\right)$, whereas Monte Carlo simulations only show a weak first-order transition. ${ }^{13}$ In fact, the Onsager theory also overvalues the coexisting number densities as compared to the simulations. It turns out that Parsons' approach both narrows the miscibility gap and shifts the coexisting densities 


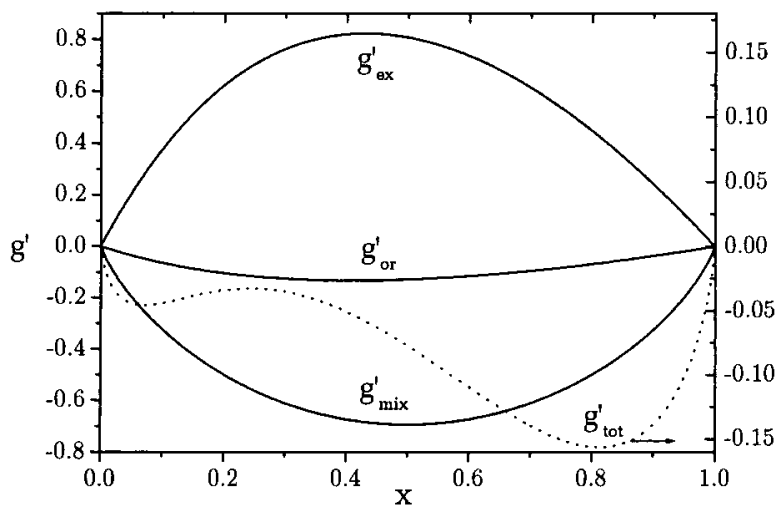

Figure 6. Rescaled Gibbs free energy $g_{\text {tot }}^{\prime}$ of the nematic phase (in units of $k_{\mathrm{B}} T$ per particle) versus mole fraction $x$ of a binary mixture of thin platelets, $D / L_{1}=13$, and thick platelets, $D / L_{2}=4\left(L_{2} / L_{1}=3.25\right)$, at coexistence pressure $\tilde{\Pi}=140$ (dotted curve). The solid curves depict the contributions from the individual entropies: excluded volume $\left(g_{\mathrm{ex}}^{\prime}\right)$, orientation $\left(g_{\mathrm{or}}^{\prime}\right)$, and mixing $\left(g_{\text {mix }}^{\prime}\right)$. The local minima in $g_{\text {tot }}^{\prime}$ indicate that the system will demix into two nematic phases.

to lower values, thus being a significant quantitative improvement over the Onsager theory. It is therefore not surprising that our preliminary calculations solely based on the Onsager theory could not establish a density inversion at any point in the phase diagram; the difference between the coexisting densities was simply too large to be overruled by fractionation, and hence an I-N density inversion was not possible.

As pointed out earlier in this paper, we intend to compare our results with the experimental observations obtained by van der Kooij. ${ }^{8}$ For this purpose, we have drawn a particular dilution line in Figure 4 which should resemble the experimental observations in a reasonable way. This "experimental" dilution line corresponds to a mole fraction $x$ of 0.07 . When a dilute system is concentrated along this dilution line, phase separation starts to occur at an overall volume fraction $\phi$ of 0.305 . The slope of the inital tie line is positive, indicating that an isotropic upper phase is formed initially. At $\phi=0.32$ the dilution line and the equal density tie line intersect, indicating that both phases are equally dense at that point. At higher volume fractions, the slopes of the tie lines become negative, indicating that the densities of the phases are inverted and a nematic upper phase will be formed. At $\phi=0.351$ the system is fully nematic. The experimental phase boundaries are found to be somewhat lower $(\phi=0.18$ and $\phi=0.30) .{ }^{8}$ Equal phase densities are found at a volume fraction of approximately 0.24 .

We finally discuss a peculiar observation in relation with the aforementioned dilution experiments. Van der Kooij performed an additional fractionation experiment in which a suspension was brought to a volume fraction $(\phi=0.29)$ close to the nematic phase boundary $(\phi=0.30)$ and left to phase separate. The nematic upper phase was separated from the isotropic bottom phase and subsequently diluted. A remarkable observation was that this system did not exhibit a density inversion at any point in the isotropic-nematic coexistence region. This striking observation however cannot be explained on the basis of our model for a binary mixture of thin and thick platelets. Figure 4 clearly shows that any dilution line close to the experimental dilution line will inevitably intersect the horizontal tie line denoting equal phase densities. This means that, according to our phase diagram, splitting off the nematic phase from a system close to the nematic phase boundary will always give rise to density inversion after dilution. In fact, an infinitesimally small dilution of an isolated upper nematic phase infinitesimally close to the phase boundary should always result in an isotropic phase with the same composition as before, even in the case of a polydisperse system. Therefore, if the fractionation experiment would be performed with infinitesimally small dilutions (which is obviously not feasible), the inverted state would inevitably be recovered after dilution of the isolated nematic phase. We therefore believe that the experimental observation can be accounted for by considering the following: First, the gap between the sample volume fraction and the phase boundary is not infinitesimally small $(\Delta \phi \approx 0.01)$, and consequently, the discarded isotropic phase has a finite volume. Second, the fractionation is believed to be particularly strong, such that practically all thick species will accumulate in the isotropic phase (with a finite volume), leaving a nematic phase with reduced polydispersity in which no density inversion can be accomplished after dilution.

We believe that the particularly strong tendency to fractionate is a manifestation of the polydisperse nature of a colloidal system of gibbsite platelets, meaning that the system essentially comprises infinitely many platelike species with continuous variations in thickness (and diameter), rather than a number of distinct species. Obviously, mapping a truly polydisperse system like this onto a simple binary model system implies a serious simplification. However, we believe that our model, despite its simplicity, is capable of capturing the essential features of the phase behavior of colloidal platelets with polydispersity in thickness.

In addition to the inversion of phase densities at the $\mathrm{I}-\mathrm{N}$ transition, our calculations show that the nematic phase of a binary mixture of thin and thick platelets can split into two nematic phases at sufficiently high concentrations. The $\mathrm{N}-\mathrm{N}$ demixing is essentially driven by the relatively small $O(L / D)$ contribution to the excluded volume part of the free energy. At sufficiently high osmotic pressures, the gain in excluded volume entropy will outweigh the loss of mixing and orientational entropy due to demixing and demixing occurs. At high thickness ratios $\left(L_{2} / L_{1} \gtrsim 3.3\right)$, an additional triphasic equilibrium is found at which two nematic phases coexist with an isotropic phase. In theoretical and simulation studies, demixing of the nematic phase into two separate nematic phases of differing densities and compositions has been found for binary mixtures of short and long rods, ${ }^{18,19}$ for thick and thin rods, ${ }^{9-11,29}$ and for rods differing in both length and diameter. ${ }^{30}$ However, no experimental efforts have been made so far to study the phase behavior of a binary mixture of thin and thick hard platelets. Considering the results of our present calculations, we believe it is a future challenge for experimentalists to verify the possibility of a nematic-nematic demixing transition in real (colloidal) binary systems.

Acknowledgment. We thank Patrick Warren for helpful discussions.

\section{References and Notes}

(1) Onsager, L. Ann. N. Y. Acad. Sci. 1949, 51, 627.

(2) Bernal, J. D.; Fankuchen, I. J. Gen. Physiol. 1941, 25, 111.

(3) Langmuir, I. J. Chem. Phys. 1938, 6, 873.

(4) Zocher, H. Z. Anorg. Allg. Chem. 1925, 147, 91.

(5) van der Kooij, F. M.; Lekkerkerker, H. N. W. J. Phys. Chem. B 1998, 102, 7829.

(6) Lekkerkerker, H. N. W.; Coulon, P.; van der Hagen, R.; Deblieck, R. J. Chem. Phys. 1984, 80, 3427.

(7) Birshtein, T. M.; Kolegov, B. I.; Pryamitsin, V. A. Polym. Sci. USSR 1988, 30, 316.

(8) van der Kooij, F. M.; van der Beek, D.; Lekkerkerker, H. N. W. J. Phys. Chem. B. 2001, 105, 1696.

(9) van Roij, R.; Mulder, B. Phys. Rev. E 1996, 54, 6430.

(10) Dijkstra, M.; van Roij, R. Phys. Rev. E 1997, 56, 5594.

(11) van Roij, R.; Mulder, B.; Dijkstra, M. Physica A 1998, 261, 374.

(12) Hemmer, P. C. J. Stat. Phys. 2000, 100, 3. 
(13) Veerman, J. A. C.; Frenkel, D. Phys. Rev. A 1992, 45, 5632.

(14) Parsons, J. D. Phys. Rev. A 1979, 19, 1225.

(15) Camp, P. J.; Allen, M. P. Physica A 1996, 229, 410.

(16) Camp, P.; Allen, M. P.; Bolhuis, P. G.; Frenkel, D. J. Chem. Phys. 1997, 106, 9270.

(17) Odijk, T.; Lekkerkerker, H. N. W. J. Phys. Chem. 1985, 89, 2090.

(18) Vroege, G. J.; Lekkerkerker, H. N. W. J. Phys. Chem. B 1993, 97, 3601.

(19) van Roij, R.; Mulder, B. J. Chem. Phys. 1996, 105, 11237.

(20) Gradshteyn, I. S.; Ryzhik, I. M. Table of Integrals, Series and Products; Academic Press: San Diego, 1994.

(21) Lee, S. D. J. Chem. Phys. 1989, 89, 7036.

(22) Lee, S. D. J. Chem. Phys. 1987, 87, 4972.

(23) Frenkel, D. J. Phys. Chem. 1988, 92, 3280.
(24) Hansen, J. P.; McDonald, I. R. Theory of Simple Liquids; Academic Press: London, 1986.

(25) Vroege, G. J.; Lekkerkerker, H. N. W. Rep. Prog. Phys. 1992, 55, 1241

(26) van der Kooij, F. M.; Lekkerkerker, H. N. W. Phys. Rev. Lett. 2000, 84,781 .

(27) van der Kooij, F. M.; Lekkerkerker, H. N. W. Langmuir 2000, 16, 10144.

(28) van der Kooij, F. M.; Vogel, M.; Lekkerkerker, H. N. W. Phys. Rev. E 2000, 62, 5397.

(29) Sear, R.; Jackson, G. J. Chem. Phys. 1995, 103, 8684.

(30) Hemmer, P. C. Mol. Phys. 1999, 96, 1153.

(31) van der Kooij, F. M. Personal communication. 